\title{
Utility of ultrasound shear wave elastography for diagnosis of malignant thyroid nodules: efficacy of standard deviation elasticity values
}

Hye Jeong Kim, Mi Kyung Kwak, Hyeong Kyu Park, Dong Won Byun, Kyoil Suh, Myung Hi Yoo

Division of Endocrinology and Metabolism, Department of Internal Medicine, Soonchunhyang University Hospital, Soonchunhyang University College of Medicine

\section{INTRODUCTION}

Shear wave elastography (SWE) is an emerging technique that can be used to evaluate malignancy in many organs. Recently, some studies have focused on providing the efficacy and diagnostic accuracy of SWE in the differential diagnosis of benign and malignant thyroid nodules, but the results showed different SWE parameters with different cut-off values. The aim of this study was to address the role of elasticity indices as a possible predictive marker for detecting papillary thyroid carcinoma (PTC) and quantitative assessment of SWE for differential diagnosis of benign and malignant thyroid nodules.

\section{METHODS}

Retrospective analysis of patients with thyroid nodules undergoing SWE before ultrasound (US)-fine needle aspiration (FNA) were analyzed. The SWE elasticity indices of mean $\left(E_{\text {Mean }}\right)$, minimum $\left(E_{\text {Min }}\right)$, maximum $\left(E_{\text {Max }}\right)$ and its standard deviation $\left(E_{S D}\right)$ of nodules were measured.

Scatter dot plots of shear wave elastography measurements for benign nodules $(n=91)$ and papillary thyroid carcinomas $(n=14)$.

(A) mean elasticity ( $\left.E_{\text {Mean }}\right)$

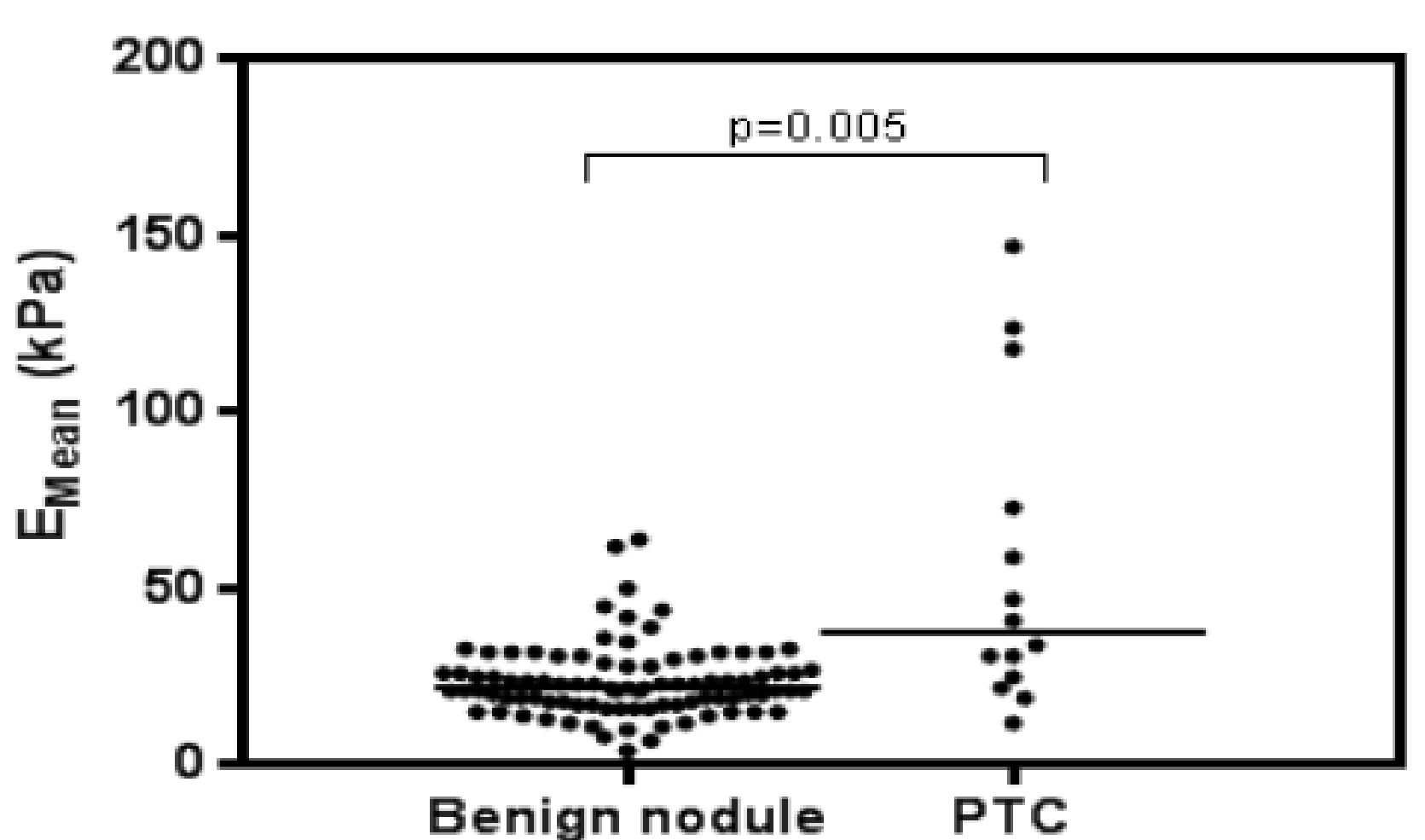

(C) maximum elasticity $\left(E_{\operatorname{Max}}\right)$

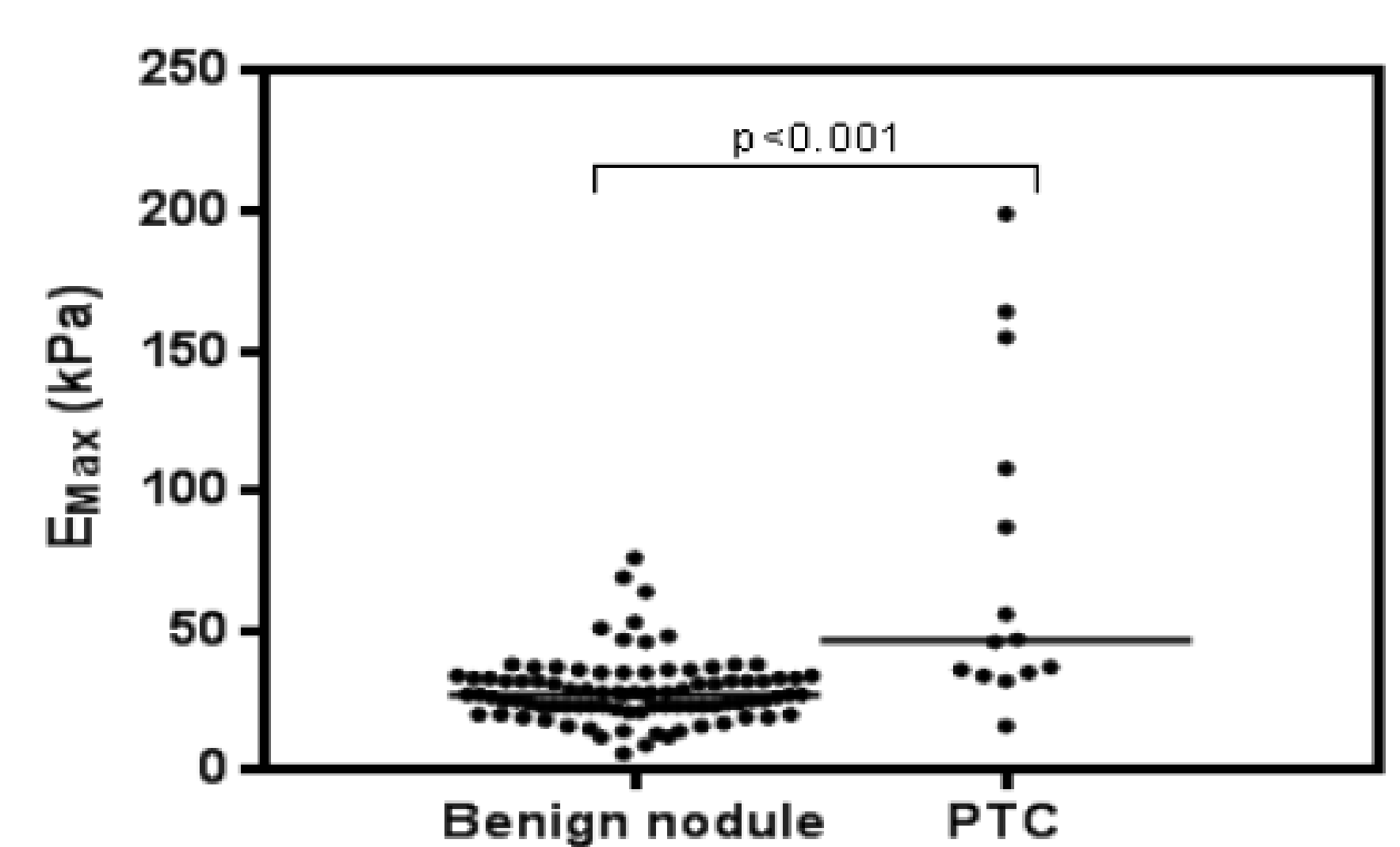

kPa, kilo-Pascal; Cental bars denote median.
(B) minimum elasticity $\left(E_{\min }\right)$

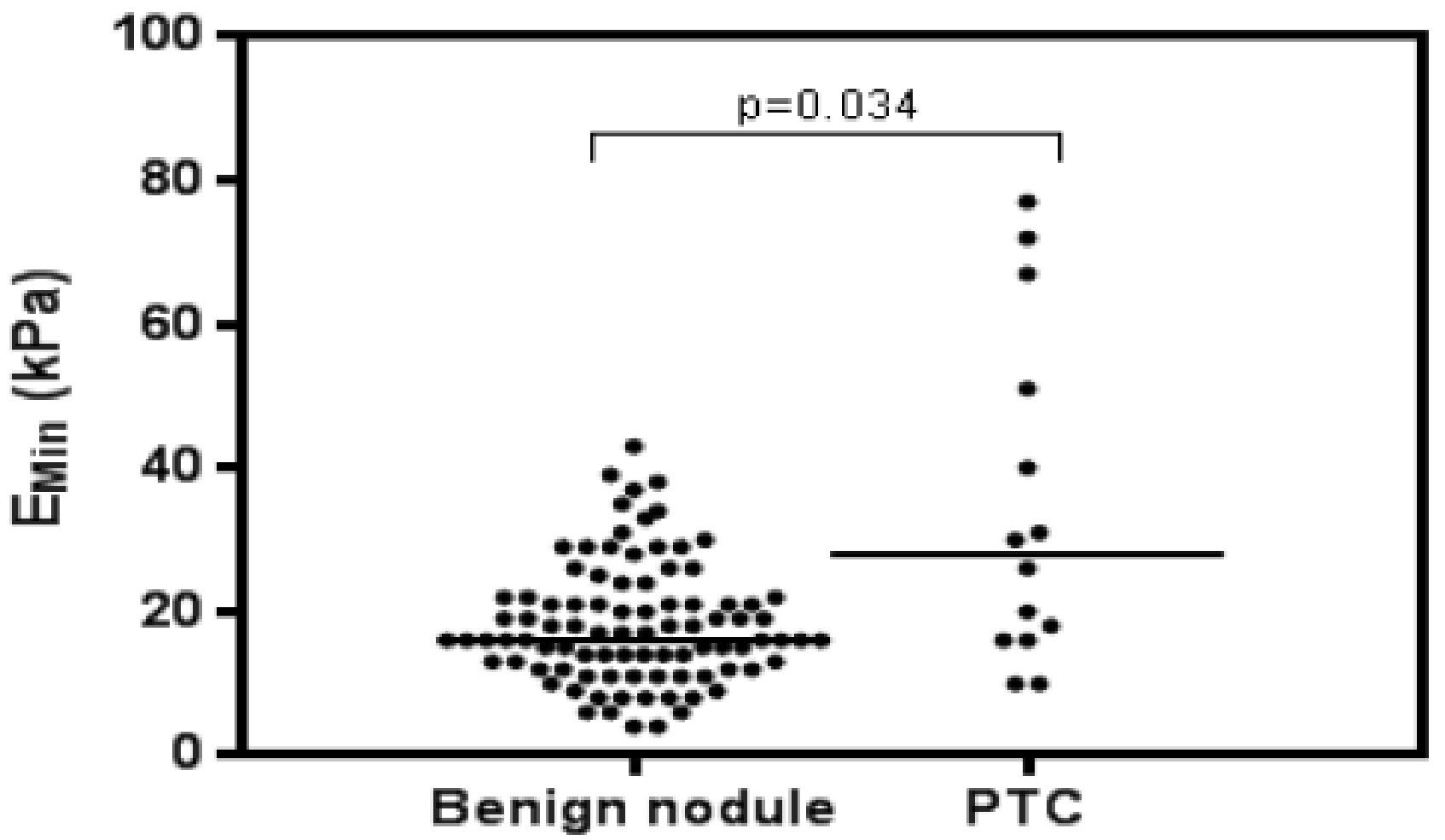

(D) one standard deviation of elastographic values $\left(E_{\mathrm{Sn}}\right)$

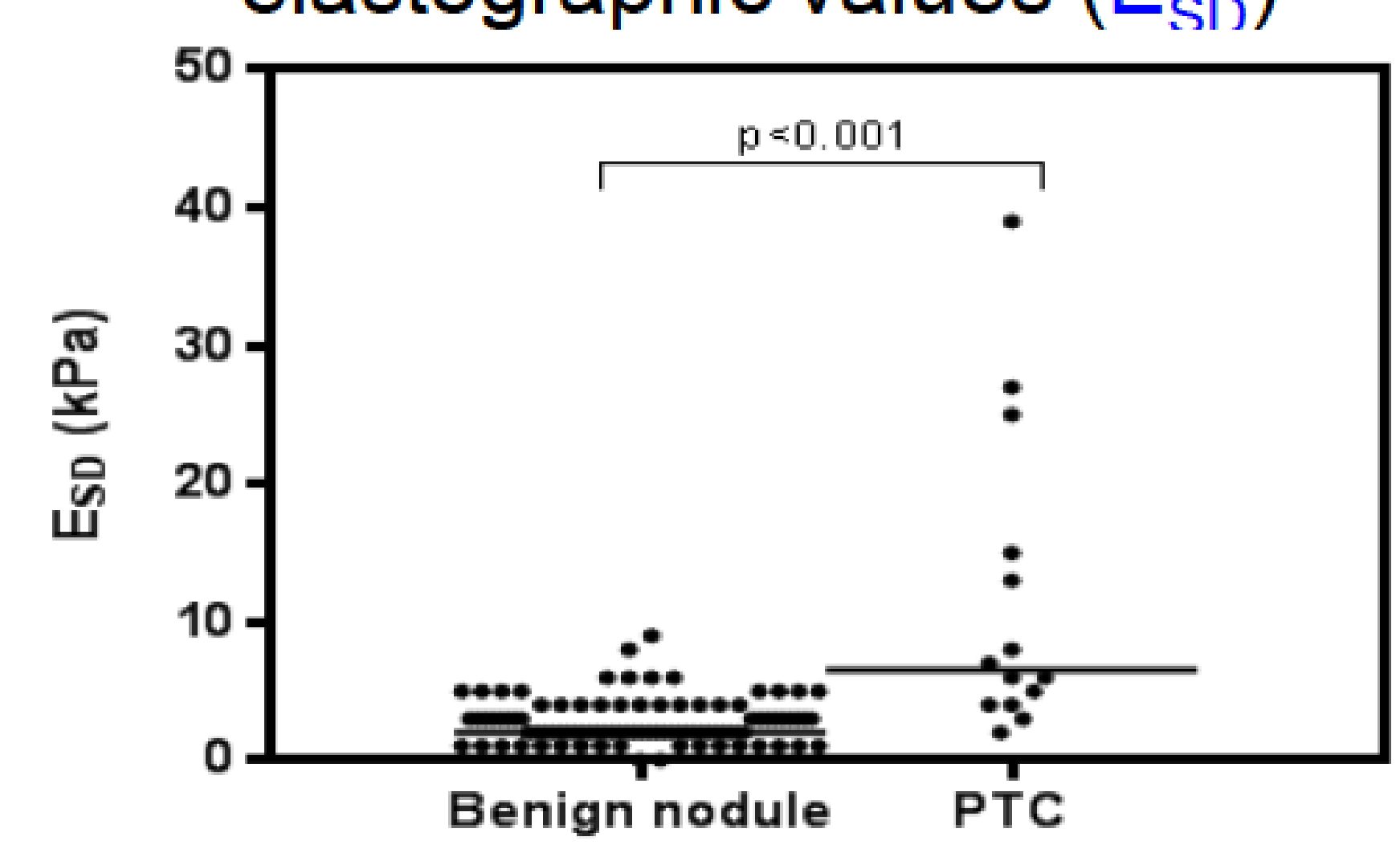

\section{RESULTS}

Shear wave elastography (upper image) and gray-scale ultrasound (lower image) of a papillary thyroid carcinoma.

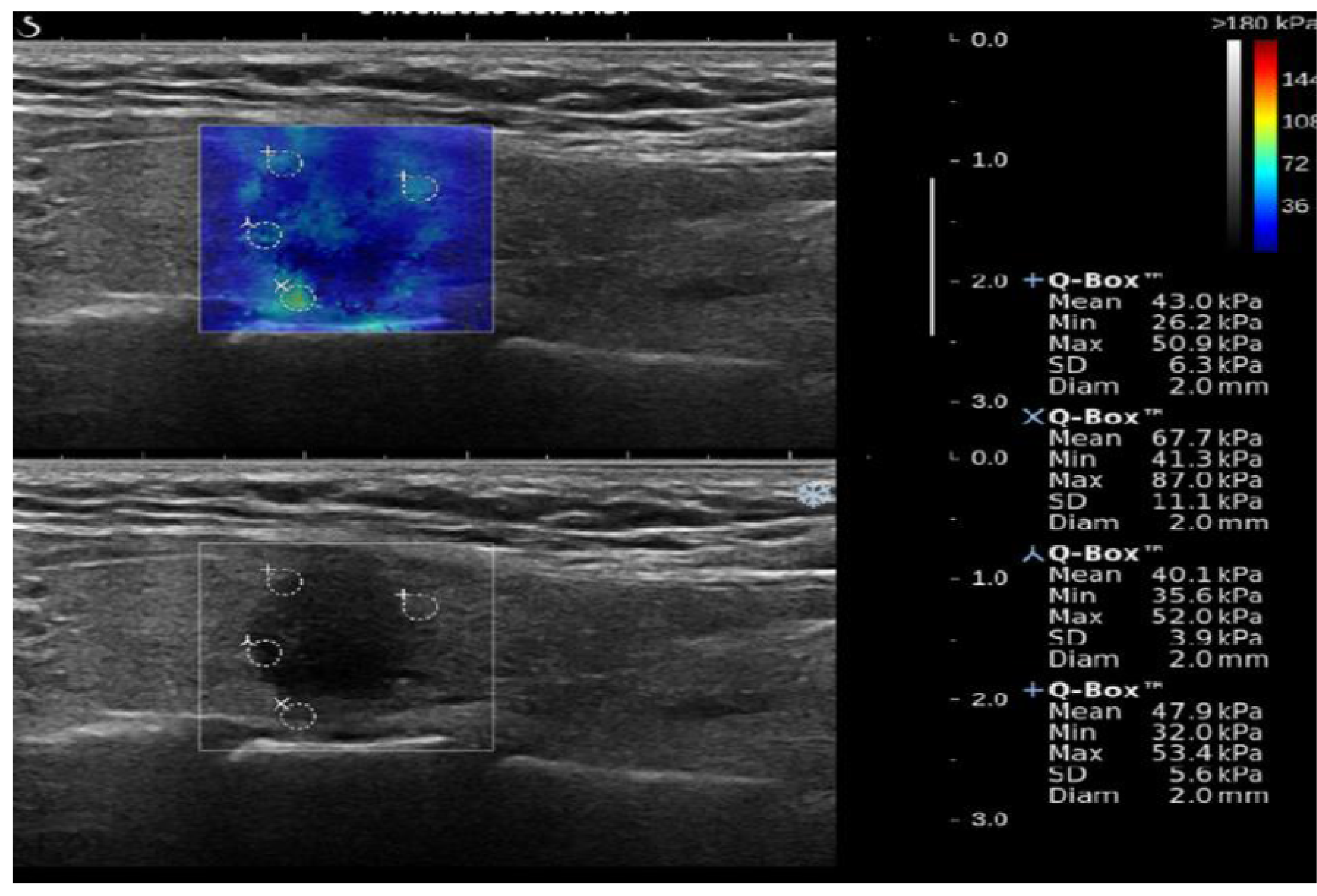

Comparison of baseline characteristics between benign nodule and papillary thyroid carcinoma $(\mathrm{N}=105)$

\begin{tabular}{lccc}
\hline Characteristic & $\begin{array}{c}\text { Benign nodule } \\
(\mathbf{n = 9 1 )}\end{array}$ & $\begin{array}{c}\text { PTC } \\
(\mathbf{n = 1 4 )}\end{array}$ & p value \\
\hline Female & $68(75 \%)$ & $10(71 \%)$ & 0.752 \\
Age (years) & $55(44,65)$ & $51(49,55)$ & 0.177 \\
Hypoechogenicity & $34(37 \%)$ & $12(86 \%)$ & 0.001 \\
Non-parallel orientation & $5(5 \%)$ & $6(43 \%)$ & 0.001 \\
Irregular margin & $15(17 \%)$ & $10(71 \%)$ & $<0.001$ \\
Microcalcification & $4(4 \%)$ & $4(29 \%)$ & 0.038 \\
Elasticity indices (kPa) & & & \\
$\mathrm{E}_{\text {Mean }}$ & $23.7(16.9,31.5)$ & $37.4(24.1,84.7)$ & 0.005 \\
$\mathrm{E}_{\text {Min }}$ & $17.8(13.4,26.4)$ & $27.9(15.9,55.1)$ & 0.034 \\
$\mathrm{E}_{\text {Max }}$ & $31.5(23.0,36.3)$ & $46.7(35.0,119.7)$ & $<0.001$ \\
$\mathrm{E}_{\mathrm{SD}}$ & $2.6(1.8,4.1)$ & $6.3(4.1,17.3)$ & $<0.001$ \\
TSH at baseline (mU/L) & $1.4(0.8,2.4)$ & $1.8(1.7,2.8)$ & 0.241 \\
\hline
\end{tabular}

PTC, papillary thyroid carcinoma; $\mathrm{kPa}$, kilo-Pascal; $\mathrm{E}_{\text {Mean, }}$ mean elasticity; $\mathrm{E}_{\text {Min }}$, minimum elasticity; $\mathrm{E}_{\mathrm{Max}}$, maximum elasticity; $\mathrm{E}_{\mathrm{SD}}$, one standard deviation of elastographic values.
ROC results and diagnostic performance of elasticity indices for predicting papillary thyroid carcinoma.

\begin{tabular}{|c|c|c|c|c|c|c|c|c|}
\hline $\begin{array}{l}\text { Elasticity } \\
\text { indices }\end{array}$ & $\begin{array}{l}\text { Cut-off } \\
\text { (kPa) }\end{array}$ & $\begin{array}{c}\text { AUC } \\
(95 \% \mathrm{Cl})\end{array}$ & $\begin{array}{c}\text { Sensitivity } \\
\text { (\%) }\end{array}$ & $\begin{array}{c}\text { Specificity } \\
\text { (\%) }\end{array}$ & $\begin{array}{c}\text { Accuracy } \\
(\%)\end{array}$ & $\begin{array}{l}\text { PLR } \\
(\%)\end{array}$ & $\begin{array}{c}\text { NLR } \\
(\%)\end{array}$ & $\begin{array}{c}\text { Diagnostic } \\
\text { odds ratio } \\
(95 \% \mathrm{Cl})\end{array}$ \\
\hline $\mathrm{E}_{\text {Mean }}$ & 33.3 & $\begin{array}{c}0.743 \\
(0.582-0.904)\end{array}$ & 57.1 & 86.4 & 80.8 & 4.21 & 0.50 & $\begin{array}{c}8.50 \\
(2.33-31.02)\end{array}$ \\
\hline$E_{\text {Min }}$ & 29.7 & $\begin{array}{c}0.683 \\
(0.508-0.858)\end{array}$ & 50.0 & 86.4 & 79.5 & 3.69 & 0.57 & $\begin{array}{c}6.74 \\
(1.76-23.06)\end{array}$ \\
\hline $\mathrm{E}_{\mathrm{Max}}$ & 45.9 & $\begin{array}{c}0.803 \\
(0.664-0.942)\end{array}$ & 57.1 & 88.1 & 82.2 & 4.82 & 0.49 & $\begin{array}{c}9.91 \\
(2.65-37.09)\end{array}$ \\
\hline$E_{S D}$ & 6.5 & $\begin{array}{c}0.849 \\
(0.723-0.975)\end{array}$ & 50.0 & 96.6 & 87.7 & 14.75 & 0.52 & $\begin{array}{c}28.50 \\
(4.92-165.09)\end{array}$ \\
\hline
\end{tabular}

$\mathrm{kPa}$, kilo-Pascal; AUC, area under the ROC curves; $\mathrm{Cl}$, confidence intervals; PLR, positive likelihood ratio; NLR, negative likelihood ratio; $E_{M a n}$ mean elasticity index; $E_{M i n}$ minimum elasticity index; $E_{M a x}$ maximum elasticity index; $E_{S D}$, standard deviation of elastographic values.

Discrimination of papillary thyroid carcinoma from benign nodules using different cut-offs.

\begin{tabular}{cccccc}
\hline $\begin{array}{c}\text { Elasticity } \\
\text { indices }\end{array}$ & $\begin{array}{c}\text { Cut-off } \\
(\mathbf{k P a})\end{array}$ & $\begin{array}{c}\text { Sensitivity (\%) } \\
\mathbf{( 9 5 \%} \mathbf{~ C l )}\end{array}$ & $\begin{array}{c}\text { Specificity (\%) } \\
\mathbf{( 9 5 \% ~ C l )}\end{array}$ & PLR & NLR \\
\hline $\mathrm{E}_{\mathrm{SD}}$ & 1.4 & $100.0(78.5-100.0)$ & $18.6(10.7-30.4)$ & 1.23 & 0.00 \\
& 2.7 & $92.8(68.5-98.7)$ & $52.5(40.4-63.9)$ & 1.96 & 0.14 \\
& 5.0 & $71.4(45.4-88.3)$ & $88.1(75.0-94.8)$ & 6.02 & 0.32 \\
& $6.5^{\mathrm{a}}$ & $50.0(26.8-73.2)$ & $96.6(88.5-99.1)$ & 14.75 & 0.52 \\
& 10.8 & $35.7(16.3-61.2)$ & $100.0(93.9-100.0)$ & - & 0.64 \\
\hline
\end{tabular}

aThreshold with highest accuracy.

\section{CONCLUSION}

The shear elasticity index of $\mathrm{E}_{\mathrm{SD}}$ with higher likelihood ratios of PTC will probably identify nodules with an increased risk for malignancy. It may help identify and select malignant nodules and also reduce unnecessary FNA of benign nodules.

\section{REFERENCES}

1. Sebag F et al. $2010 \mathrm{~J}$ Clin Endocrinol Metab 95:5281-5288

2. Veyrieres JB et al. 2012 Eur J Radiol 81:3965-3972.

3. Bhatia KS et al. 2012 Eur Radiol 22:2397-2406. 